identify the antimicrobial agents involved in bacterial resistance.

Methods A cross-sectional study, with data obtained retrospectively through medical records review. We analysed 140 patients whose women's endocervical samples and men's urine tested positive for $\mathrm{MH}$ and UU. The test used for the diagnosis and evaluation of antimicrobial resistance was the MYCOFAST Screening EvolutioN 3 kit.

Results The majority of patients were female (90.7\%), 57.9\% of the patients had $>29$ years of age and $84.3 \%$ did not have a stable union. About $70.0 \%$ of the patients tested positive for UU, $3.0 \%$ for $\mathrm{MH}$, and $27.0 \%$ for both. HIV coinfection was seen in $32.1 \%$ and HPV in $30.0 \%$. Absence of current history of Chlamydia trachomatis was a protective factor for the acquisition of azithromycin resistance $(p=0.04)$. As for the antimicrobials, doxycycline showed sensitivity rates higher than $96 \%$ for both infections, while azithromycin showed $86.8 \%$ of sensitivity for UU, but resistance of $75 \%$ for $\mathrm{MH}$. Ciprofloxacin showed sensitivity rates lower than $15 \%$ for both infections, while less than 35\% were strains sensible to ofloxacin. Erythromycin resistance rates ranged over $65 \%$ for the UU-MH coinfection, while over $90 \%$ of sample was sensible to tetracycline and clarithromycin resistance rates ranged from $7.1 \%$ for UU to $100 \%$ for $\mathrm{MH}$.

Conclusion The use of ciprofloxacin and ofloxacin is highly debatable considering the high rates of total and intermediate resistance. In our population, doxycycline showed high efficiency and is therefore recommended for the treatment of UU and $\mathrm{MH}$ infections. Monitoring antimicrobial resistance is fundamental for the adequacy of the therapeutic recommendations.

\section{P3.84 ESTIMATING THE ANTIBODY PREVALENCE OF HERPES SIMPLEX VIRUS TYPE 1 AMONG SELECT MIDDLE EAST AND NORTH AFRICA POPULATIONS}

'Gheyath Nasrallah, ${ }^{2}$ Soha Dargham, 'Layla Mohammed, '2Laith Abu-Raddad. 'Qatar University, Doha, Qatar; ${ }^{2}$ Weill Cornell Medicine-Qatar, Doha, Qatar

\section{$10.1136 /$ sextrans-2017-053264.319}

Introduction Evidence indicates a growing role for herpes simplex virus type-1 (HSV-1) as a sexually transmitted infection and as the etiological agent of genital herpes, but HSV-1 epidemiology in the Middle East and North Africa (MENA) remains poorly understood. We aimed to measure HSV-1 antibody prevalence among select MENA populations and to characterise the infection's age-distribution.

Methods Evidence indicates a growing role for herpes simplex virus type-1 (HSV-1) as a sexually transmitted infection and as the etiological agent of genital herpes, but HSV-1 epidemiology in the Middle East and North Africa (MENA) remains poorly understood. We aimed to measure HSV-1 antibody prevalence among select MENA populations and to characterise the infection's age-distribution.

Results Country-specific HSV-1 prevalence was estimated for 10 MENA national populations of male blood donors residing in Qatar. HSV-1 prevalence was estimated at 97.5\% $(95 \%$ confidence interval (CI) 95.3\%-98.7\%) among Egyptians, 92.6\% (95\% CI 87.2\%-95.8\%) among Yemenis, 90.7\% (95\% CI 84.5\%-94.6\%) among Sudanese, 88.5\% (95\% CI 83.9\%92.6\%) among Syrians, 86.5\% (95\% CI 81.0\%-90.5\%) among
Jordanians, $82.3 \%$ (95\% CI 78.2\%-85.7\%) among Qataris, $81.4 \%$ (95\% CI 73.3\%-87.5\%) among Iranians, 81.4\% (95\% CI 73.4\%-87.4\%) among Lebanese, $80.5 \%$ (95\% CI $74.2 \%$ $85.2 \%$ ) among Palestinians, and $77.0 \%$ (95\% CI $70.7 \%$ 82.3\%) among Pakistanis. Age-specific HSV-1 prevalence was estimated among male blood donors from Egypt, the Fertile Crescent (Iraq, Jordan, Lebanon, Palestine and Syria) and Qatar. There was a trend of growing HSV-1 prevalence with age among the Fertile Crescent and Qatari nationals. HSV-1 prevalence increased from 70.0\% (95\% CI 56.3\%-80.9\%) for those aged $<24$ years up to $98.0 \%$ (95\% CI $89.5 \%-99.7 \%$ ) for those aged $\geq 55$ years in the Fertile Crescent. Similar pattern was observed for Qatar, but for Egypt, prevalence was steadily above $90 \%$ for all age groups. Our results showed no significant association between sex and HSV-1 seropositivity.

Conclusion HSV-1 prevalence in MENA continues at high level, but for the majority of nationalities, at considerably lower levels than historical levels. The decline in prevalence is most pronounced among youth. As much as a third of those $<30$ years of age are reaching sexual debut with no protective antibodies against HSV-1 genital acquisition, and accordingly, are at risk of genital herpes.

\section{P3.85 EVALUATION OF GENTAMICIN SUSCEPTIBILITY AND RESISTANCE PHENOTYPES OF NEISSERIA GONORRHOEAE ISOLATES IN ARGENTINA}

${ }^{1} \mathrm{R}$ Gianecini, ${ }^{1} \mathrm{C}$ Oviedo, ${ }^{2} \mathrm{GASSP}-\mathrm{AR},{ }^{1}$ Galarza P. ${ }^{1}$ Servicio de Enfermedades de Transmisión Sexual, Instituto Nacional de Enfermedades Infecciosas (INEI)-ANLIS "Dr. Carlos G. Malbrán", Ciudad Autónoma de Buenos Aires, Argentina.; 'Gonococcal Antimicrobial Susceptibility Surveillance Programme-Argentina (GASSP-AR)

\subsection{6/sextrans-2017-053264.320}

Introduction The emergence of multidrug resistance in N. gonorrhoeae highlights the need to consider alternatives for future therapeutic use. Nowadays, the aminoglycoside gentamicin is considered a possible treatment option for gonorrhoea, particularly in dual therapy. Gentamicin has been used successfully for several years in the treatment of gonococcal urethritis in Malawi. However, treatment failures have been reported. In Argentina, N. gonorrhoeae isolates with multiple resistance profiles to different antimicrobials, including cefixime and ceftriaxone, have been detected. Therefore, the knowledge of the susceptibility to new antimicrobial options for gonorrhoea treatment is required. The aim of this study was to determine the susceptibility to gentamicin of $N$. gonorrhoeae isolates with different resistance profiles in 2013 and 2015.

Methods We studied 237 N. gonorrhoeae isolates obtained in 2013 and 2015 from the GASSP-AR. The MICs to penicillin, tetracycline, ciprofloxacin, cefixime, ceftriaxone, azithromycin and gentamicin were determined by the agar dilution test according to CLSI guidelines.

Results Gentamicin MICs ranged from 2 to $16 \mu \mathrm{g} / \mathrm{ml}$. The $\mathrm{MIC}_{50}$ and $\mathrm{MIC}_{90}$ were $8 \mu \mathrm{g} / \mathrm{ml}$. The rate of isolates with MIC $8 \mu \mathrm{g} / \mathrm{ml}$ was $67.1 \%$, while $30.4 \%$ showed a MIC of $4 \mu \mathrm{g} / \mathrm{ml}$. A $75.5 \%$ of the isolates included in this study showed resistance to one or more of the following antibiotics: penicillin (40.5\%), tetracycline (30.4\%), ciprofloxacin $(59.9 \%)$, and $5.5 \%$ of the isolates showed decreased susceptibility to cefixime and ceftriaxone. A total of 15 resistance phenotypes 
were found, with phenotypes QRNG and PPNG being more frequent.

Conclusion Although N. gonorrhoeae isolates with resistance to gentamicin were not observed, a high percentage of strains were resistant to other antimicrobial agents, particularly ciprofloxacin. This study suggests that gentamicin may be considered a future treatment option for gonorrhoea in Argentina. However, the high prevalence of isolates with MIC $8 \mu \mathrm{g} / \mathrm{ml}$ (intermediate susceptibility) suggests a continuous surveillance of gentamicin in our country.

\section{P3.86 COMPARATIVE EVALUATION OF DISK DIFFUSION AND AGAR DILUTION METHODS FOR GENTAMICIN SUSCEPTIBILITY TESTING OF NEISSERIA GONORRHOEAE}

${ }^{1} \mathrm{R}$ Gianecini, ${ }^{1} \mathrm{C}$ Oviedo, ${ }^{2} \mathrm{~L}$ Irazu, ${ }^{2} \mathrm{M}$ Rodríguez, ${ }^{3} \mathrm{G}$ ASSP-AR, ${ }^{1}$ Galarza P. ${ }^{1}$ Servicio de Enfermedades de Transmisión Sexual, Instituto Nacional de Enfermedades Infecciosas (INEI)ANLIS "Dr. Carlos G. Malbrán", Ciudad Autónoma de Buenos Aires, Argentina.; ${ }^{2}$ Departamento de Parasitología, Instituto Nacional de Enfermedades Infecciosas (INEI)ANLIS "Dr. Carlos G. Malbrán", Ciudad Autónoma de Buenos Aires, Argentina; ${ }^{3}$ Gonococcal Antimicrobial Susceptibility Surveillance Programme-Argentina (GASSP-AR)

\subsection{6/sextrans-2017-053264.32}

Introduction At present, gentamicin is a promising antibiotic for the treatment of multidrug resistant $N$. gonorrhoeae isolates. Therefore, the knowledge of N. gonorrhoeae susceptibility to gentamicin is required. The CLSI guidelines do not list breakpoints for gentamicin. However, MIC interpretive criteria have been proposed. Moreover, a recent report comparing the disk diffusion with Etest has established tentative gentamicin zone breakpoints for the CLSI method. The proposed breakpoints are $\geq 16 \mathrm{~mm}$ for susceptible, $13-15 \mathrm{~mm}$ for intermediate and $\leq 12 \mathrm{~mm}$ for resistant. The aim of this study was to compare the disk diffusion method with the agar dilution test, and to analyse the suitability and reliably of disk diffusion to monitor the susceptibility to gentamicin.

Methods We studied $237 \mathrm{n}$. gonorrhoeae isolates obtained in 2013 and 2015 from the GASSP-AR. The MIC determination and disk diffusion tests to gentamicin were performed according to CLSI, and tentative breakpoints previously reported were used. The 2008 WHO and ATCC 49226 reference strains were used as control. The inhibition diameters by disk diffusion were tested by correlation with the MIC value.

Results Gentamicin MICs ranged from 2 to $16 \mu \mathrm{g} / \mathrm{ml}$, and the MIC $_{50 / 90}$ were both $8 \mu \mathrm{g} / \mathrm{ml}$. The Pearson correlation between disk diffusion and agar dilution was $-0.67 \quad(\mathrm{p}<0.001)$. No very major or major discrepancies were detected with disk diffusion as compared to agar dilution. However, a high percentage of minor discrepancies (39.9\%) was observed. By adjusting the susceptible breakpoint for disk diffusion to $S \geq 17$ $\mathrm{mm}$, the minor discrepancies rate was reduced from $39.9 \%$ to $18.4 \%$.

Conclusion: N. gonorrhoeae isolates with resistance to gentamicin were not observed. The disk diffusion had good correlation when compared with the agar dilution method. Although a high percentage of minor discrepancies was observed, the error rate was reduced adjusting the breakpoint. Until it becomes standardised, the disk diffusion can be a screening method in clinical laboratories to detect the gentamicin susceptibility of N. gonorrhoeae.

\section{P3.87 ASYMPTOMATIC URETHRITIS PREVALENCE AND ASSOCIATED RISK FACTORS AMONG MALES LIVING WITH HIV-1}

Guilherme Almeida Rosa da Silva. UNIRIO, Rio De Janeiro - RJ, Brazil

\subsection{6/sextrans-2017-053264.322}

Introduction It is estimated that symptomatic carriers of $N$. gonorrhoeae and C. trachomatis have a relative risk of 4.8 fold and 3.6 fold, respectively, for the sexual acquisition of HIV. This type of evaluation for asymptomatic urethritis is necessary to reinforce strategies to combat HIV transmission.

Methods We enrolled a total of 115 male patients aged 18 years or older who have been diagnosed with an HIV infection and have no symptoms of urethritis or other sexually transmitted infections. Urine was collected and tested by realtime PCR for the detection of C. trachomatis and N. gonorrhoeae (Abbott RealTime CT/NG test ${ }^{\circledR}$ ). Simultaneous clinical data and blood collection was performed to obtain HIV viral load values and $\mathrm{CD} 4^{+} \mathrm{T}$ lymphocyte counts.

Results Four patients had asymptomatic carrier status of urethritis and were positive for C. trachomatis. The prevalence was $3.47 \%$. Patients who were positive for C. trachomatis urethritis had a lower mean age $(\mathrm{p}=0.015)$. After statistical analysis, we observe that age is associated with the time of use of ART $\left(\beta=\right.$ positive, $\left.\mathrm{R}^{2}=0.082, \mathrm{p}<0.01\right)$ and that the mean age of patients who do not use condoms is higher than those who use condoms in more than or equal to $50 \%$ of the relationships $(p=0.03)$. Likewise, the mean age in patients who use condoms in less than $50 \%$ of relationships is higher than that of patients who use condoms in more than or equal to $50 \%$ of relationships $(p=0.04)$. The status of single men is associated with sexual intercourse with other men or men and women and condom use in more than 50\% of relationships $(\mathrm{p}<0.01)$. Uncircumcised men are associated with the highest plasma HIV viral load among patients with detectable HIV load when compared to circumcised men $(\mathrm{p}<0.01)$.

Conclusion We recommend that, in outpatient practice, the habit of inquiring about history of sexual behaviour to obtain more information about risks and associations with asymptomatic sexually transmitted infection, a routine physical examination and complementary tests for the agents should be discarded.

\section{P3.88 FREQUENCY OF SYPHILIS TESTING AMONG MEN WHO HAVE HAD RECTAL TESTS FOR CHLAMYDIA AND GONORRHOEA, UNITED STATES}

${ }^{1}$ Guoyu Tao, ${ }^{1}$ Thomas Peterman, ${ }^{2}$ Melinda B Nye, ${ }^{1}$ Thomas L Gift, ${ }^{2}$ Barbara A Body. ${ }^{1} C D C$, Atlanta, USA; ${ }^{2}$ LABCORP, Burlington, USA

\subsection{6/sextrans-2017-053264.323}

Introduction CDC recommends syphilis screening at least annually for sexually active MSM and screening every 3-6 months for MSM with risks such as multiple partners.

Methods In collaboration with a large U.S. commercial laboratory, we identified men aged 15-60 years who had rectal chlamydia (CT) or gonorrhoea (GC) testing during September, 2013-Augest, 2015 as presumed MSM. We estimated: the frequency of testing (counting only tests $\geq 3$ months after a previous test); reactivity of nontreponemal tests (NTT) and treponemal tests (TT); and the association between CT/GC and the reactivity of syphilis NTT and TT. We also identified 